\title{
Room-Temperature Topological Phase Transition in Quasi-One-Dimensional Material $\mathrm{Bi}_{4} \mathbf{I}_{4}$
}

\author{
Jianwei Huang $\odot,{ }^{1, *}$ Sheng $\mathrm{Li},{ }^{2, *}$ Chiho Yoon $\odot,{ }^{2,3, *}$ Ji Seop Oh $\odot,{ }^{4,1} \mathrm{Han} \mathrm{Wu},{ }^{1}$ Xiaoyuan Liu, ${ }^{2}$ Nikhil Dhale, ${ }^{2}$ \\ Yan-Feng Zhou, ${ }^{2}$ Yucheng Guo $\odot,{ }^{1}$ Yichen Zhang, ${ }^{1}$ Makoto Hashimoto, ${ }^{5}$ Donghui Lu, ${ }^{5}$ Jonathan Denlinger, \\ Xiqu Wang, ${ }^{7}$ Chun Ning Lau, ${ }^{8}$ Robert J. Birgeneau $\odot,{ }^{4,9, \dagger}$ Fan Zhang $\oplus^{2, \$}$ Bing Lv, ${ }^{2,8}$ and Ming Yi® ${ }^{1, \|}$ \\ ${ }^{1}$ Department of Physics and Astronomy, Rice University, Houston, Texas 77005, USA \\ ${ }^{2}$ Department of Physics, The University of Texas at Dallas, \\ Richardson, Texas 75080-3021, USA \\ ${ }^{3}$ Department of Physics and Astronomy, Seoul National University, Seoul 08826, Korea \\ ${ }^{4}$ Department of Physics, University of California, Berkeley, California 94720, USA \\ ${ }^{5}$ Stanford Synchrotron Radiation Lightsource, SLAC National Accelerator Laboratory, \\ Menlo Park, California 94025, USA \\ ${ }^{6}$ Advanced Light Source, Lawrence Berkeley National Laboratory, Berkeley, California 94720, USA \\ ${ }^{7}$ Department of Chemistry, University of Houston, Houston, Texas 77204, USA \\ ${ }^{8}$ Department of Physics, The Ohio State University, Columbus, Ohio 43210, USA \\ ${ }^{9}$ Materials Science Division, Lawrence Berkeley National Laboratory, Berkeley, California 94720, USA
}

(Received 7 February 2021; revised 30 April 2021; accepted 27 May 2021; published 24 August 2021)

Quasi-one-dimensional (1D) materials provide a superior platform for characterizing and tuning topological phases for two reasons: (i) existence for multiple cleavable surfaces that enables better experimental identification of topological classification and (ii) stronger response to perturbations such as strain for tuning topological phases compared to higher dimensional crystal structures. In this paper, we present experimental evidence for a room-temperature topological phase transition in the quasi-1D material $\mathrm{Bi}_{4} \mathrm{I}_{4}$, mediated via a first-order structural transition between two distinct stacking orders of the weakly coupled chains. Using high-resolution angle-resolved photoemission spectroscopy on the two natural cleavable surfaces, we identify the high-temperature $\beta$ phase to be the first weak topological insulator with two gapless Dirac cones on the (100) surface and no Dirac crossing on the (001) surface, while in the lowtemperature $\alpha$ phase, the topological surface state on the (100) surface opens a gap, consistent with a recent theoretical prediction of a higher-order topological insulator beyond the scope of the established topological materials databases that hosts gapless hinge states. Our results not only identify a rare topological phase transition between first-order and second-order topological insulators but also establish a novel quasi-1D material platform for exploring unprecedented physics.

DOI: 10.1103/PhysRevX.11.031042

\section{INTRODUCTION}

Topological phases and associated phase transitions in quantum materials have garnered tremendous interest in recent years since the discovery of the two-dimensional (2D) quantum spin Hall effect [1,2]. Extending this concept to three dimensions (3D) led to the idea of topological

\footnotetext{
*These authors contributed equally to this work. †robertjb@berkeley.edu

zhang@utdallas.edu

§blv@utdallas.edu

"mingyi@rice.edu
}

Published by the American Physical Society under the terms of the Creative Commons Attribution 4.0 International license. Further distribution of this work must maintain attribution to the author(s) and the published article's title, journal citation, and DOI.
Subject Areas: Condensed Matter Physics

insulator (TI), which hosts gapless boundary states. Within the class of TIs, there are two schemes by which the topological class can be further distinguished. The first scheme is the distinction between strong and weak TIs $[3,4]$. While every surface of a strong TI possesses a gapless Dirac cone, such nontrivial surface states only appear on selected surfaces of a weak TI [4-7]. The experimental identification between a strong and a weak TIs therefore depends critically on the measurement of topological nontrivial surface states on multiple surfaces of a material, commonly probed via the technique of angleresolved photoemission spectroscopy (ARPES). The challenge of such experimental efforts is that in natural materials usually only a single preferred cleavage surface is experimentally accessible for ARPES. Most TIs that have been experimentally confirmed are strong TIs, identified by a combination of a single-surface measurement and theoretical calculations, such as in the bismuth and antimony 


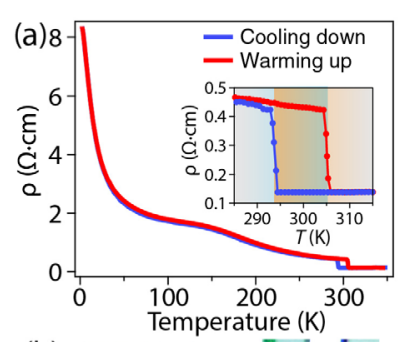

(b)

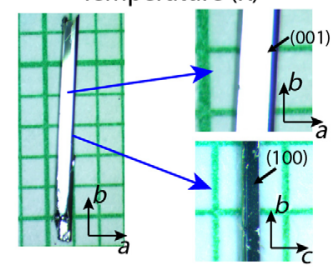

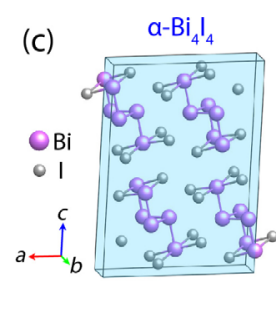
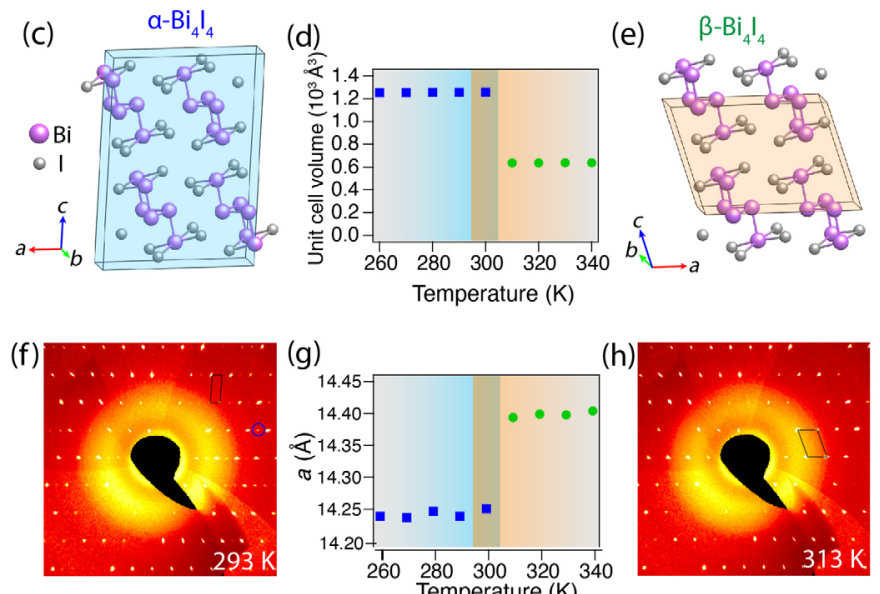

(g)

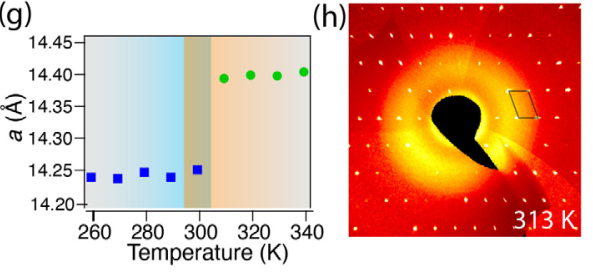

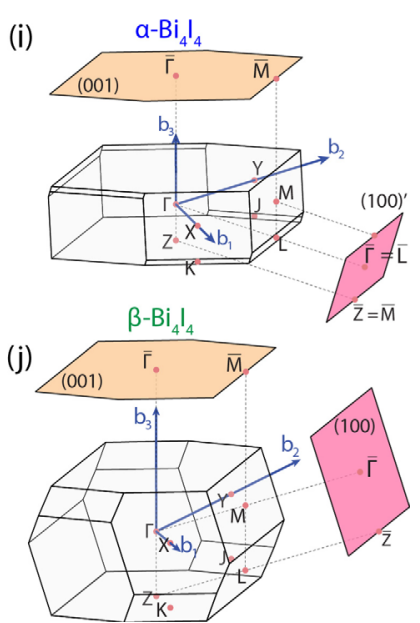

FIG. 1. Crystal structure and sample characterization. (a) Temperature-dependent resistivity data for both cooling and warming curves. The inset is the enlarged view showing the hysteresis behavior of the first-order transition. (b) $\operatorname{Images}$ of a $\mathrm{Bi}_{4} \mathrm{I}_{4}$ crystal showing the cleavable (001) and (100) surfaces where the grid is $1 \mathrm{~mm}$. (c),(f) The real-space crystal structure and the reciprocal space lattice from the $h 0 l$ layer precession images integrated from $\mathrm{x}$-ray diffraction data for the $\alpha-\mathrm{Bi}_{4} \mathrm{I}_{4}$ phase. The shaded region is the structural unit cell. (d),(g) The unit cell volume and refined lattice parameter $a$ from x-ray diffraction reflecting the $\alpha$ to $\beta$ structural transition. (e),(h) Same as (c),(f) but for the $\beta-\mathrm{Bi}_{4} \mathrm{I}_{4}$ phase. (i),(j) The bulk and projected surface Brillouin zones (BZ) of $\alpha-\mathrm{Bi}_{4} \mathrm{I}_{4}$ and $\beta$ - $\mathrm{Bi}_{4} \mathrm{I}_{4}$.

chalcogenides [8-11]. Direct experimental verification of a weak TI, however, remains scarce due to the lack of materials with multiple cleavage surfaces where the existence of topologically nontrivial surface states could be probed $[7,12-17]$. The second scheme to classify further a TI is by its topological order, which is distinguished by the dimension of its gapless boundary states compared to the dimension of its bulk state. This realization led to the identification of a novel class of phases of matter dubbed the higher-order TI [18-21], which are materials that host protected gapless states on boundaries of two or three spatial dimensions lower than its bulk.

Interestingly, materials predicted to be candidate weak TIs are often in the vicinity of a topological critical point, where the exact topological classification depends sensitively upon the lattice parameters $[7,22]$. On the one hand, this produces an uncertainty in theoretical predictions of their topological properties, which makes experimental determination a necessity $[7,22]$. On the other hand, this renders an advantage that a topological phase transition between different topological phases can be easily induced by external perturbations such as strain and thermal effects [7,22-24].

As a quasi-1D material, $\mathrm{Bi}_{4} \mathrm{I}_{4}$ provides a superior platform not only to realize but also to be experimentally examined for the aforementioned distinct TI classes [7,21]. Consisting of stacked quasi-1D chains, $\mathrm{Bi}_{4} \mathrm{I}_{4}$ naturally stabilizes in two crystal structures due to distinct stacking sequences, identified as $\alpha-\mathrm{Bi}_{4} \mathrm{I}_{4}$ and $\beta-\mathrm{Bi}_{4} \mathrm{I}_{4}$ [25]. The $\alpha$ phase is naturally stabilized at low temperatures and transitions into the $\beta$ phase via a first-order phase transition around $300 \mathrm{~K} . \beta-\mathrm{Bi}_{4} \mathrm{I}_{4}$ was theoretically predicted to be topologically nontrivial, in the vicinity of a transition point between weak and strong TIs [7,26]. Two subsequent ARPES studies reached conflicting conclusions [26,27]. While one study reports strong TI characteristics for a lowtemperature phase identified as $\beta-\mathrm{Bi}_{4} \mathrm{I}_{4}$ [26], the other utilized a quenching method to stabilize the $\beta-\mathrm{Bi}_{4} \mathrm{I}_{4}$ phase at low temperatures and identified it as a weak TI [27] while it categorized the $\alpha-\mathrm{Bi}_{4} \mathrm{I}_{4}$ phase as a trivial insulator with a distinct electronic structure [27]. Besides the controversy among the experimental reports, these results also differ from a recent theoretical work predicting the $\alpha$ phase to be a topologically nontrivial higher-order TI hosting helical hinge modes [21]. While beyond the scope of the recently established topological materials database [28-30], this predicted rare phase may be viewed as a quantum spin Hall effect $[1,2]$ around the top or bottom surface of a 3D insulator, i.e., the time-reversal-invariant counterpart of the long-desired 3D quantum Hall effect [31,32]. To resolve the aforementioned controversies, we systematically carried out electrical transport, $\mathrm{x}$-ray diffraction, and ARPES measurements in combination with theoretical calculations to clarify the topological classification of $\alpha-\mathrm{Bi}_{4} \mathrm{I}_{4}$ and $\beta-\mathrm{Bi}_{4} \mathrm{I}_{4}$ as tuned by the natural structural transition via temperature.

\section{STRUCTURAL TRANSITION BETWEEN THE $\alpha$ AND $\beta$ PHASES}

Large $\mathrm{Bi}_{4} \mathrm{I}_{4}$ single crystals were synthesized with the quasi-1D chains oriented along the $b$ axis, with large flat (001) and (100) surfaces (Fig. 1) (see Supplemental Material [33]). The structural phase transition can be clearly identified from the temperature-dependent resistivity [Fig. 1(a)] and heat capacity (Supplemental Material 
Fig. S1 [33]) measurements, where a hysteresis indicating a first-order phase transition appears around $300 \mathrm{~K}$. Below $270 \mathrm{~K}$, the resistivity data show a typical semiconductor behavior with a broad hump at $150 \mathrm{~K}$ and are consistent with previous reports [27].

Structurally, the $\alpha$ and $\beta$ phases differ in the packing of the $\mathrm{Bi}_{4} \mathrm{I}_{4}$ chains. The $\alpha-\mathrm{Bi}_{4} \mathrm{I}_{4}$ has double-layered $\mathrm{Bi}_{4} \mathrm{I}_{4}$ chains per unit cell [Fig. 1(c)] whereas the $\beta-\mathrm{Bi}_{4} \mathrm{I}_{4}$ has only a single layer of $\mathrm{Bi}_{4} \mathrm{I}_{4}$ chains per unit cell [Fig. 1(e)]. This subtle difference in stacking causes a large change of the $c$ lattice and the associated doubling of the unit cell volume [Fig. 1(d)], as demonstrated by X-ray single crystal diffraction [Figs. 1(f) and 1(h)]. Contrasting the single crystal diffraction patterns for the reciprocal lattice $a^{*}-c^{*}$ planes taken in the $\alpha$ phase at $293 \mathrm{~K}$ [Fig. 1(f)] and the $\beta$ phase at $313 \mathrm{~K}$ [Fig. 1(h)], a near doubling of the $c^{*}$ in reciprocal space from $\alpha-\mathrm{Bi}_{4} \mathrm{I}_{4}$ to $\beta-\mathrm{Bi}_{4} \mathrm{I}_{4}$ is visible, as seen by the additional peaks in Fig. 1(f) marked by a circle. This structural change is well captured in the temperaturedependent refined lattice parameters [Fig. 1(g) and Supplemental Material Fig. S2 [33] ]. The lattice parameters of the $\alpha$ phase are refined at $300 \mathrm{~K}$ to be $a=14.251(2) \AA$, $b=4.4304(5) \AA, c=19.976(6) \AA$, and $\beta=92.93(2)^{\circ}$, while those of the $\beta$ phase are refined at $310 \mathrm{~K}$ to be $a=14.394(5) \AA, \quad b=4.4288(13) \AA, c=10.494(5) \AA$, and $\beta=107.96(5)^{\circ}$.

The large facets on both the (001) $(>0.5 \mathrm{~mm})$ and (100) $(>0.2 \mathrm{~mm})$ surfaces are naturally cleavable, exposing flat surfaces that enable conventional ARPES studies on either surface using an incident beam spot size of $50 \times 20 \mu \mathrm{m}^{2}$ [Fig. 1(b)]. We note the $\beta$-angle difference between $\alpha-\mathrm{Bi}_{4} \mathrm{I}_{4}$ and $\beta-\mathrm{Bi}_{4} \mathrm{I}_{4}$ crystal structures, where the natural cleavable side (100) surface of $\beta-\mathrm{Bi}_{4} \mathrm{I}_{4}$ becomes the (201) surface in the $\alpha$ phase per definition in the true primitive unit cell [Figs. 1(c) and 1(e)]; we label it as $(100)^{\prime}$ in $\alpha-\mathrm{Bi}_{4} \mathrm{I}_{4}$ [Figs. 1(i) and 1(j)] to avoid confusion in the comparison with the (100) surface of the $\beta$ phase.

Taking advantage of the rare multisurface experimental access to $\mathrm{Bi}_{4} \mathrm{I}_{4}$, we carried out ARPES measurements on both the (001) and (100) surfaces of the $\alpha$ and $\beta$ phases in their respective temperature regimes. We note that such two-surface ARPES measurement has also been desirable to identify unambiguously the topological characters of weak TIs and higher-order TIs. A comparison of the complete electronic structure of the two phases is summarized in Fig. 2. Two main observations can be made: (i) there is a strong distinction between the electronic structures measured on the (100) and (001) surfaces for both phases and (ii) the change between the two phases across the structural transition is subtle.

\section{DISTINGUISHING THE (001) AND (100) SURFACES}

We first examine the distinction between the (100) and (001) surfaces, which is most clearly seen in the measured
Fermi surfaces (FS). On the (100) surface, both phases exhibit a quasi-1D FS along $k_{z}$ [Figs. 2(a) and 2(c)], revealing a rather weak interlayer coupling in the $c$ direction. The FS on the (001) surface, by contrast, consists of islandlike bright spots for both phases [Figs. 2(b) and 2(d) and Supplemental Material Fig. S9 [33] ], suggesting a stronger interlayer coupling along the $a$ axis compared to that of the $c$ axis. Furthermore, the measured sample surface orientation can be unambiguously distinguished by the periodicity of the observed band dispersions, which corresponds to the clearly distinct lattice parameters associated with the (100) and (001) surfaces. The $\alpha$ phase, for example, has a period of $0.3 \AA^{-1}$ along the $k_{z}$ direction on the $(100)^{\prime}$ surface [Fig. 2(m)] and $0.88 \AA^{-1}$ along the $k_{x}$ direction on the (001) surface [Fig. 2(n)]. We note that we do not observe a drastic change of the fermiology across the phase transition, in contrast to the previous report that the quasi-1D FS only exists in the $\beta$ phase [27]. By clearly identifying single domains of each cleaved surface, we now clarify that the quasi-1D intensity and islandlike intensity are due to the two different crystal surfaces measured rather than the two distinct structural phases.

We can furthermore examine the corresponding lowenergy band dispersions. At the high symmetry points of both surfaces in both phases, an intense holelike bulk band is observed below $E_{F}$, revealing a gap in the bulk state in all cases. The bulk nature of this band is confirmed by photon energy dependence as it disperses along $k_{z}$ (Fig. S6 [33]). However, two observations can be noted. Firstly, as the electronic structure is quasi-1D along $k_{z}$ on the (100) surfaces, the bands near the $\bar{\Gamma}$ and $\bar{Z}$ points are quite similar in both phases [Figs. 2(e), 2(f), 2(i), and 2(j)] while those near the $\bar{\Gamma}$ and $\bar{M}$ points on the (001) surface are notably distinct. Secondly, while we observe a holelike bulk valence band on all the cuts shown, within the bulk band gap on the (100) surface, a sharp Dirac surface state is observed for both the $\alpha$ and $\beta$ phases [Figs. 2(e), 2(f), 2(i), and 2(j)]. On the contrary, no Dirac surface state is observed inside the bulk band gap on the (001) surface of either phase [Figs. 2(g), 2(h), 2(k), and 2(l)]. At the $\bar{\Gamma}$ point, the bulk valence band tops at $-0.3 \mathrm{eV}$. No surface state is observed. At the $\bar{M}$ point, the bulk valence band approaches $E_{F}$, with a visible gap near $E_{F}$ between the bulk valence and conduction bands.

\section{ELECTRONIC RECONSTRUCTION BETWEEN THE $\alpha$ AND $\beta$ PHASES}

Having distinguished the two surfaces, we now focus on the transition between the two structural phases. Overall, no dramatic change of the bulk band dispersions is observed across the phase transition. There is, however, a doubling in the number of bands from the $\beta$ phase to the $\alpha$ phase [21] due to the doubling of the stacking layers in the unit cell. This band doubling is well demonstrated by our theoretical 

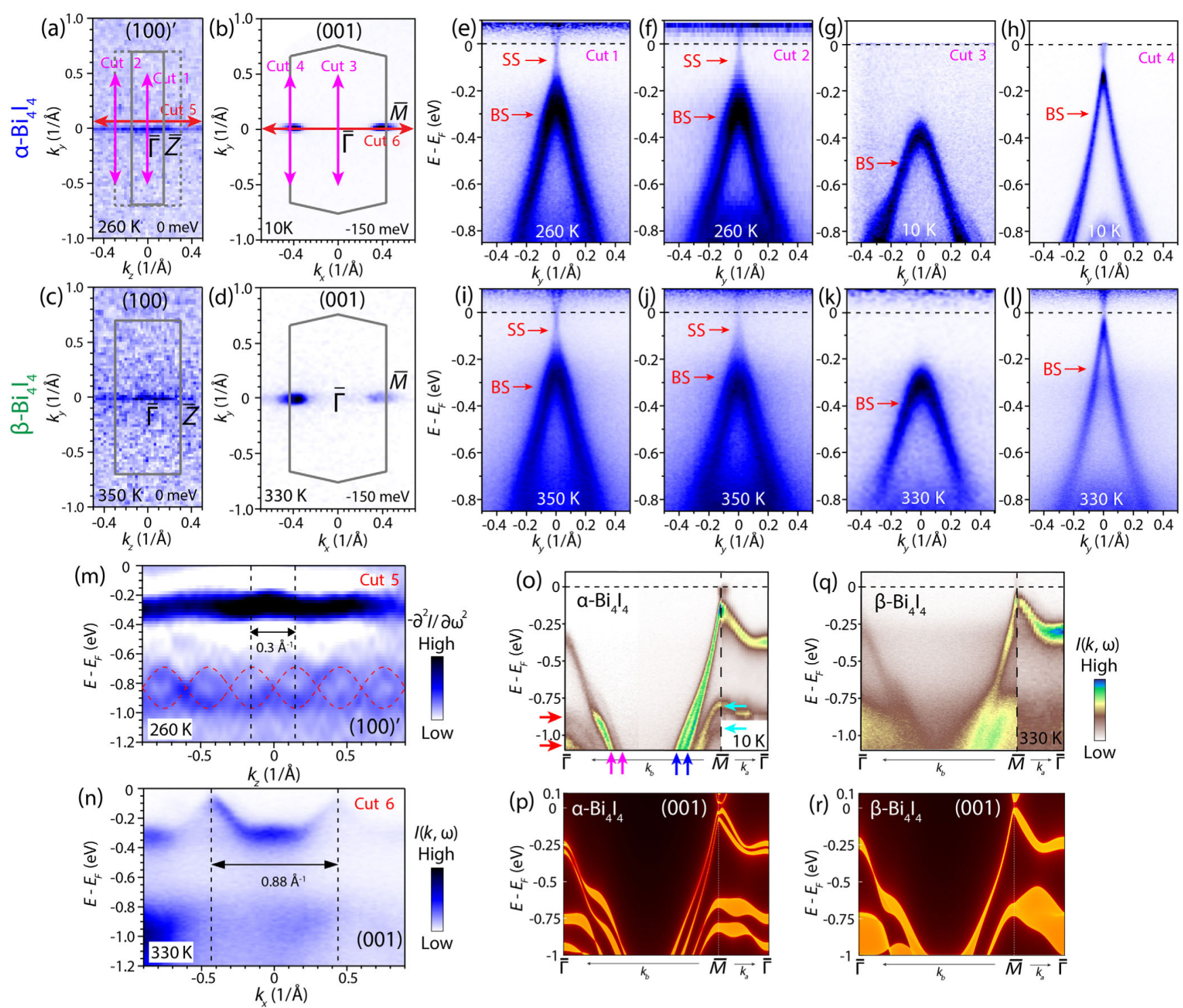

FIG. 2. Electronic structure from different surfaces. (a) Fermi surface mapping of $\alpha-\mathrm{Bi}_{4} \mathrm{I}_{4}$ on the $(100)^{\prime}$ surface, with the surface BZ marked by gray rectangles (the solid one corresponds to $\alpha-\mathrm{Bi}_{4} \mathrm{I}_{4}$ and the dashed one to $\beta$ - $\mathrm{Bi}_{4} \mathrm{I}_{4}$ ). (b) Constant energy contour of $\alpha$ - $\mathrm{Bi}_{4} \mathrm{I}_{4}$ on the (001) surface. (e)-(h) Measured band dispersions of $\alpha-\mathrm{Bi}_{4} \mathrm{I}_{4}$ along the cut 1 to cut 4 indicated in (a) and (b). Related surface states (SS) and bulk states (BS) are marked by arrows. (c),(d) and (i)-(l) Same as the top row but for $\beta$-Bi $\mathrm{I}_{4}$. All band images are divided by the Fermi-Dirac function. (m) Band image along the cut 5 except taken at $k_{y}=0$. Cut 5 was drawn intentionally off center to not block the Fermi surface image. The red dashed lines are sinusoidal fittings of the band image showing a periodicity of $0.3 \AA^{-1}$ due to band doubling, which corresponds to the $(100)^{\prime}$ surface. (n) Band image along the cut 6 indicated in (b). The black arrow shows a periodicity of $0.88 \AA^{-1}$ which corresponds to the (001) surface. (o) Measured dispersions in $\alpha-\mathrm{Bi}_{4} \mathrm{I}_{4}$ along high symmetry cuts. (p) Calculated bulk band structure of $\alpha-\mathrm{Bi}_{4} \mathrm{I}_{4}$ projected onto the (001) surface (see Supplemental Material [33]). (q), (r) Same as (o), (p) but for $\beta$-Bi ${ }_{4} \mathrm{I}_{4}$. A clear doubling of the bulk valence bands is evident from $\beta$ - $-\mathrm{Bi}_{4} \mathrm{I}_{4}$ to $\alpha-\mathrm{Bi}_{4} \mathrm{I}_{4}$ in both the measured and calculated dispersions. All measurement temperatures are as indicated.

calculations of the bulk bands projected onto the (001) surface for both the $\alpha$ and $\beta$ phases [Figs. 2(p) and 2(r) and Supplemental Material [33]). While the band splitting is small due to the weak interlayer coupling, it is clearly resolved in our corresponding dispersions measured at low temperatures in the $\alpha$ phase [see pairs of arrows in Figs. 2(o) and a larger energy window shown in
Fig. S3 [33]], consistent with the doubled stacking in the low-temperature phase.

Next, we demonstrate that this band doubling is indeed induced via the structural transition by systematically measuring across the structural transition near room temperature. We note that while it is challenging to resolve the small band splitting near the valence band top at elevated 

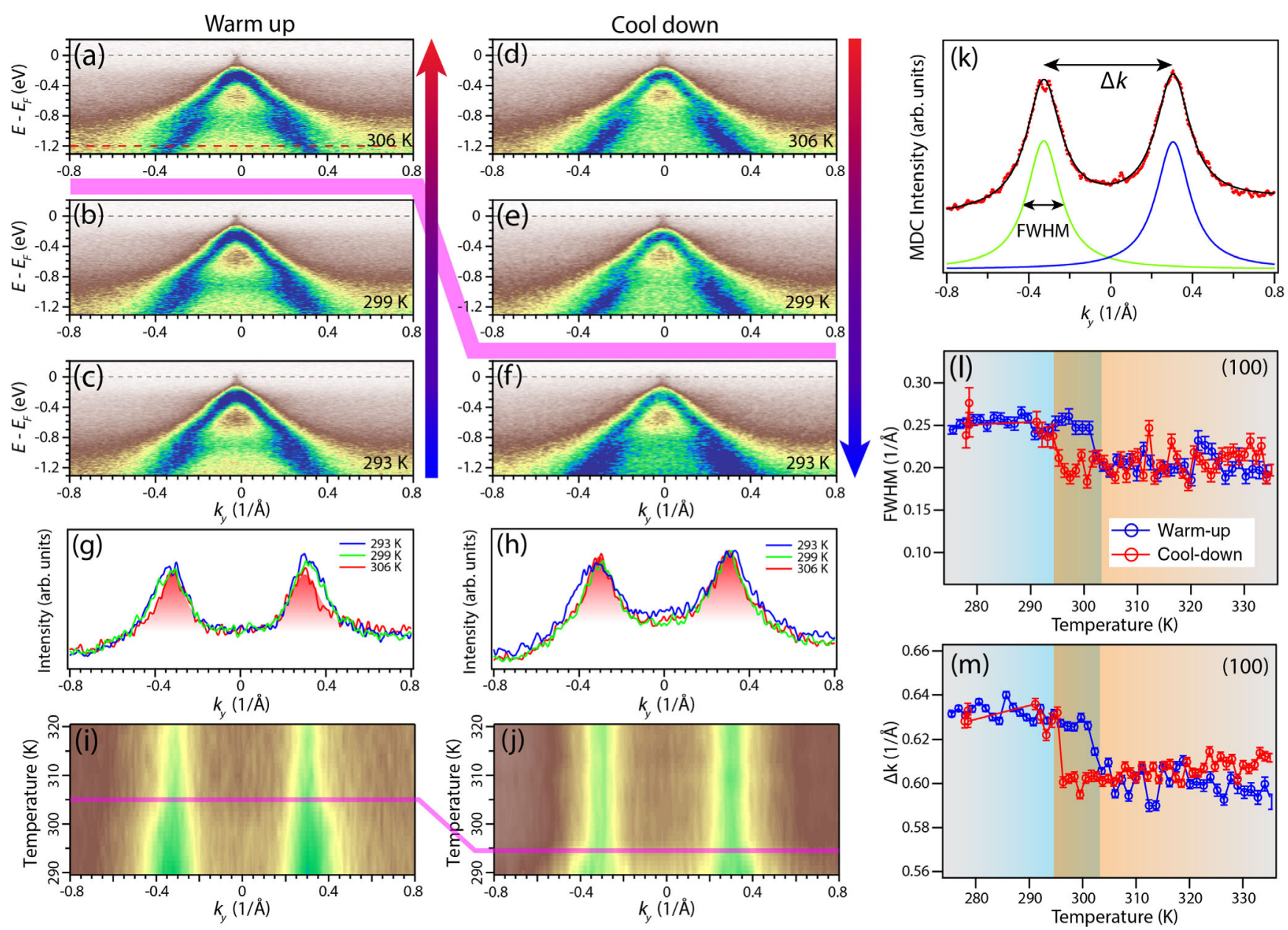

FIG. 3. Bulk band doubling across the phase transition. (a)-(c) Warm-up and (d)-(f) cool-down band images measured along cut 1 indicated in Fig. 2(a) at selected temperatures. (g), (h) Corresponding MDCs at $-1.2 \mathrm{eV}$ during warm-up and cool-down, respectively. (i) Warm-up and (j) cool-down false-color images showing a more detailed temperature evolution of the MDCs indicated in (a). (k) Example of a MDC fitted by two Lorentzian peaks. (1) Full width at half maximum of the MDC fittings indicated in (k) as a function of temperature. The warm-up results are shifted $0.04 \AA^{-1}$ upward. (m) The separation of the peak positions of the MDC fittings indicated in $(\mathrm{k})$ as a function of temperature.

temperatures, this band doubling manifests as a broadening in the momentum distribution curves (MDCs) when a single band splits into two from the higher-temperature $\beta$ phase to the lower-temperature $\alpha$ phase. Clearly, this effect is in contrast to thermal broadening effects, which would have the opposite trend with respect to temperature. In Figs. 3(a)-3(c), we plot the dispersions across the $\bar{\Gamma}$ point measured on (100) at selected temperatures. A clear abrupt broadening is indeed observed from the hightemperature $\beta$ phase to the lower-temperature $\alpha$ phase as exemplified by the MDCs at $-1.2 \mathrm{eV}$ [Figs. 3(i) and $3(\mathrm{j})$ ]. Importantly, this abrupt change is accompanied by a hysteresis behavior revealed in the warm-up [Fig. 3(i)] and cool-down [Fig. 3(j)] processes. To quantify this behavior, we fit the MDCs and plot both their full width at half maximum [Fig. 3(1)] and peak positions [Fig. 3(m)], both of which show the hysteresis behavior reminiscent of that in the resistivity measurement, confirming the electronic structure's response to the firstorder structural transition.

\section{TOPOLOGICAL CHARACTERIZATION}

Having confirmed the phase transition as observed from the electronic structure, we examine the topological properties of the two phases. In Fig. 4 we present high-resolution measurements near $E_{F}$ for both phases. We first examine the $\beta$ phase. On the (001) surface [Figs. 2(k), 2(l), and 4(b)], we observe a clear gap in all the bulk gaps and no additional surface Dirac crossings. This can be confirmed from the energy distribution curve (EDC) stacks of the bands across $\bar{M}$ [Fig. 4(b)], where the green dots track the bulk band dispersion and reveal a gap of $\sim 100 \mathrm{meV}$. Similar suppressed intensity can also be clearly identified in the MDC stacks (Fig. S7 [33]). On the (100) surface, a quasi-1D Dirac surface state exists inside the bulk band gap 


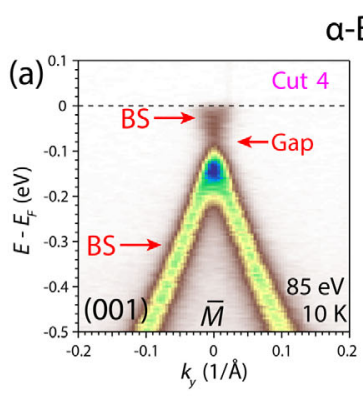

$\mathrm{a}-\mathrm{Bi}_{4} \mathrm{I}_{4}$

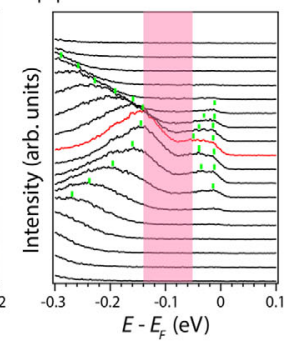

..... Bulk band ...... Surface band

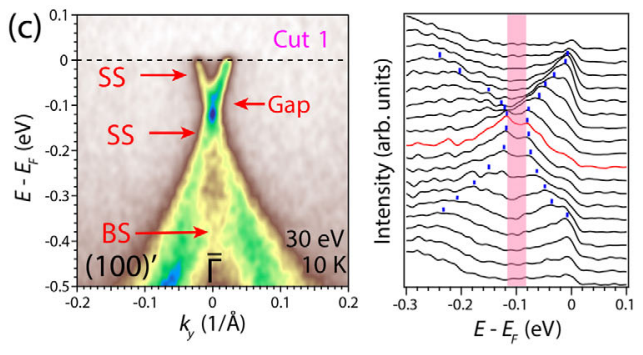

(e)

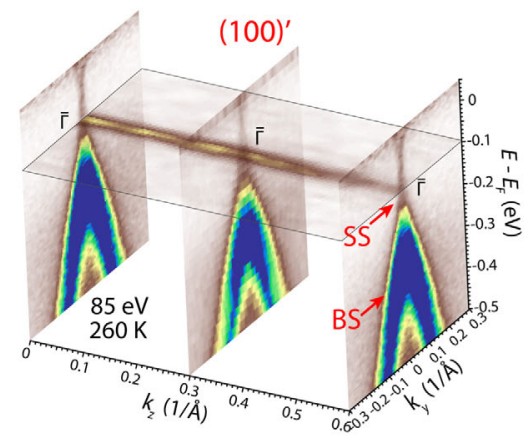

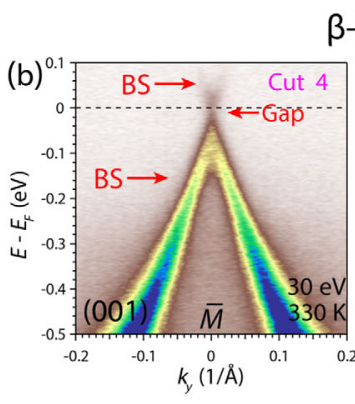

$\beta-\mathrm{Bi}_{4} \mathrm{I}_{4}$

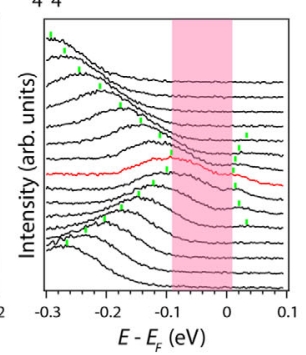

(g)

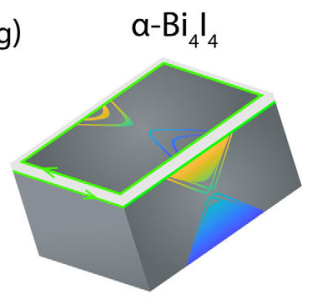

(d)
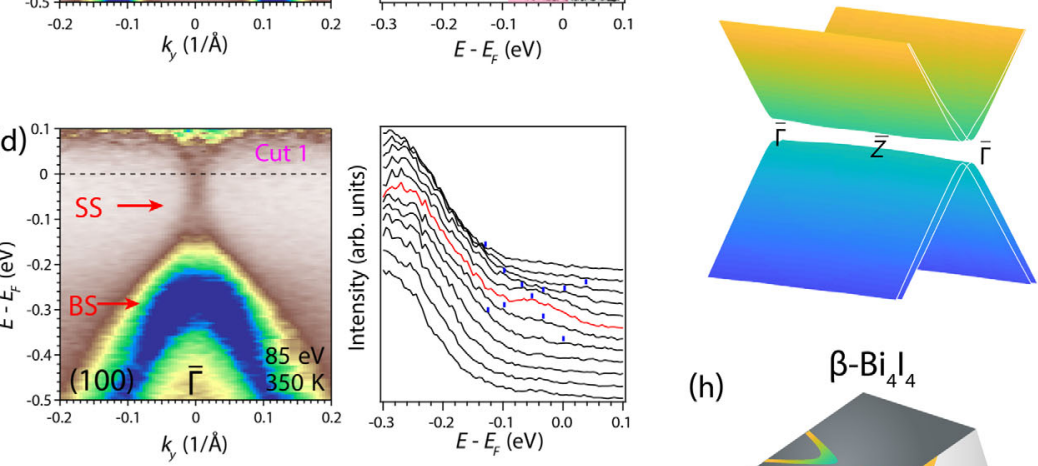

(h)

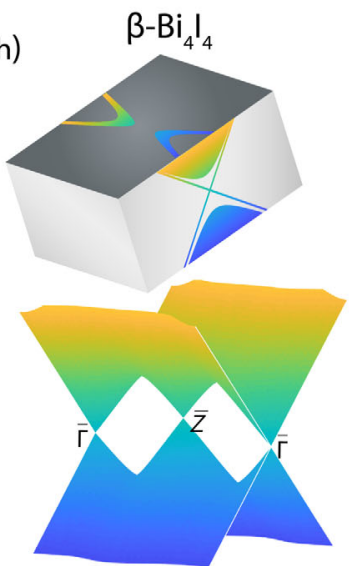

FIG. 4. Surface states evolution across the phase transition. (a) Band image along cut 4 on the (001) surface indicated in Fig. 2(b) of $\alpha-\mathrm{Bi}_{4} \mathrm{I}_{4}$ as well as its corresponding EDC stacks. The red EDC corresponds to $k=0$. The green dots track the peak positions of the bulk band which reveal a gap opening of $\sim 85 \mathrm{meV}$. (b) Same as (a) but of $\beta-\mathrm{Bi}_{4} \mathrm{I}_{4}$. A gap opens at $k=0$ of $\sim 100 \mathrm{meV}$. (c) Band image along cut 1 on the $(100)^{\prime}$ surface indicated in Fig. 2(a) of $\alpha-\mathrm{Bi}_{4} \mathrm{I}_{4}$ as well as its corresponding EDC stacks. The blue dots track the surface state which reveal a $\sim 35 \mathrm{meV}$ gap opening. (d) Same as (c) but of $\beta-\mathrm{Bi}_{4} \mathrm{I}_{4}$. A gapless Dirac surface state is observed. (e) 3D view of the electronic structure on the (100)' surface of $\alpha-\mathrm{Bi}_{4} \mathrm{I}_{4}$. A quasi-1D Dirac-like surface state is observed. (f) Same as (e) but of $\beta$-Bi $\mathrm{I}_{4}$. The quasi-1D Dirac surface state reveals the weak TI property. (g) Schematics (see Supplemental Material [33]) showing the higher-order TI state of $\alpha-\mathrm{Bi}_{4} \mathrm{I}_{4}$ with a particular surface termination. Upper panel: all the bulk states and surface states are gapped, whereas a helical hinge state around the top surface exists in the surface state gap. Lower panel: the enlarged (100) surface state features a band doubling and a small gap. (h) Schematics (see Supplemental Material [33]) showing the weak TI state of $\beta$ - $\mathrm{Bi}_{4} \mathrm{I}_{4}$. Upper panel: the bulk states and (001) surface state are gapped, whereas there is a quasi-1D gapless Dirac surface state on the (100) surface. Lower panel: the enlarged (100) surface state features two gapless Dirac cones.

[Figs. 2(i), 2(j), 4(d), and 4(f)]. Particularly at the $\bar{\Gamma}$ and $\bar{Z}$ points, the surface states are gapless within the resolution of measurements at $350 \mathrm{~K}$. These combined measurements on both the (001) and (100) surfaces demonstrate that the hightemperature $\beta-\mathrm{Bi}_{4} \mathrm{I}_{4}$ is a weak TI where only selected surfaces possess an even number (two here) of gapless Dirac cones [3,7] [Fig. 4(h)]. In the $\alpha$ phase, all the observed bands on the (001) surface are also gapped [Figs. 2(g), 2(h), and 4(a)]. A gap of $\sim 85 \mathrm{meV}$ can be further seen from the bands tracked by the EDC stacks in Fig. 4(a). On the $(100)^{\prime}$ surface, the quasi-1D Dirac-like surface state still persists [Figs. 2(e) and 2(f)]. From a high-resolution measurement taken with $30 \mathrm{eV}$ photons at $10 \mathrm{~K}$ [Fig. 4(c)], we observe a gap of $\sim 35 \mathrm{meV}$ clearly in the EDC stacks, indicating a gapped surface state within a bulk band gap. This is consistent with our theoretical prediction and calculation of a higher-order TI scenario of $\alpha-\mathrm{Bi}_{4} \mathrm{I}_{4}$ [21], illustrated in Fig. 4(g), where both the bulk bands and surface bands are required to be gapped, hosting gapless hinge states inside the surface gap. A comparison of our calculated bulk and surface states with our measured bands shows strong agreement (Fig. S10 [33]). We summarize our experimental findings and classification of the two phases in Table I. 
TABLE I. Summary of the key features on $\alpha-\mathrm{Bi}_{4} \mathrm{I}_{4}$ and $\beta-\mathrm{Bi}_{4} \mathrm{I}_{4}$ revealed by our ARPES study.

\begin{tabular}{lccccc}
\hline \hline Structure & Temperature & Bulk bands & $(001)$ surface & $(100)$ surface & Topology \\
\hline$\alpha-\mathrm{Bi}_{4} \mathrm{I}_{4}$ & $<295 \mathrm{~K}$ & $8 N$ & $\sim 85 \mathrm{meV}(\bar{M})$ & $\sim 35 \mathrm{meV}(\bar{\Gamma})$ & Higher-order TI \\
$\beta-\mathrm{Bi}_{4} \mathrm{I}_{4}$ & $>305 \mathrm{~K}$ & $4 N$ & $\sim 100 \mathrm{meV}(\bar{M})$ & Gapless & Weak TI \\
\hline \hline
\end{tabular}

Finally, we comment on the inconsistencies in the previous two ARPES studies on $\mathrm{Bi}_{4} \mathrm{I}_{4}$. In the first report [26], since no quenching was performed, the phase measured at low temperatures was most likely the $\alpha$ phase instead of the claimed $\beta$ phase. The conclusion that this phase is a strong TI was based on the observation of a gapless Dirac crossing on the (001) surface. Here we show that this Dirac band is rather a bulk band, where highresolution measurements reveal a gap [Fig. 4(a)]. In the second report with nanoARPES [27], the $\beta$ phase was claimed to be stabilized at low temperature by quenching directly from high temperature. The authors ascribed pointlike FS to $\alpha-\mathrm{Bi}_{4} \mathrm{I}_{4}$ and quasi-1D FS to $\beta-\mathrm{Bi}_{4} \mathrm{I}_{4}$ based on a superposition of signals from the (001) and (100) surfaces. Based on this assumption, they concluded that the $\beta$ phase is a weak TI and the $\alpha$ phase is a trivial insulator. Benefiting from the relatively large cleavable (100) and (001) surfaces free of domains, here we unambiguously show that both phases have similar quasi-1D FSs on the (100) side surface, and that the distinction between them is the subtle band doubling in the $\alpha$ phase, which was not resolved in previous studies. Reminiscent of the bulk Peierls distortion in the Su-Schrieffer-Heeger model [34], this yields a gap opening in the Dirac surface state on the $(100)^{\prime}$ surface, which is the prerequisite for the appearance of midgap hinge states in $\alpha-\mathrm{Bi}_{4} \mathrm{I}_{4}$ as a higher-order TI [21].

\section{DISCUSSION}

Topological properties of a variety of quantum materials have been verified by a combination of ARPES and firstprinciples calculations during the past decades $[10,11$, 35-42]. For the quasi-1D material $\mathrm{Bi}_{4} \mathrm{I}_{4}$, not only does the uncertainty of the theoretical prediction for the topological classification of both $\alpha-\mathrm{Bi}_{4} \mathrm{I}_{4}$ and $\beta-\mathrm{Bi}_{4} \mathrm{I}_{4}$ call for experimental exploration, but also the change of the electronic structures between $\beta-\mathrm{Bi}_{4} \mathrm{I}_{4}$ and $\alpha-\mathrm{Bi}_{4} \mathrm{I}_{4}$ is subtle such that careful ARPES measurements with considerable energy resolution is necessary. Unambiguously, the $\beta$-phase $\mathrm{Bi}_{4} \mathrm{I}_{4}$ is shown to be the first weak TI by our ARPES measurements on large natural cleavable planes of the top and side surfaces, which resolves the controversy of the previous reports [26,27]. For $\alpha-\mathrm{Bi}_{4} \mathrm{I}_{4}$, two distinctions are clearly observed as compared with $\beta-\mathrm{Bi}_{4} \mathrm{I}_{4}$ : band doubling and gap opening in the side surface state. Together with the identification of the $\beta$ phase as a weak TI, the existence of the Dirac-like surface state with a small gap indicates that the $\alpha$ phase is consistent with the identification as a rare higher-order TI [21] instead of the trivial insulator based on symmetry indicators alone [28-30]. Since the $\beta$ phase has been shown as a weak TI here, each (001) monolayer is a $2 \mathrm{D} Z_{2}$ TI [21]. As the $\alpha$ phase has been shown to have two monolayers per unit cell here, i.e., a $2 \mathrm{D}$ trivial insulator, the $3 \mathrm{D}$ bulk must be $Z_{2}$ trivial given the weak interlayer couplings [3,7]; in other words, the surface states are gapped. Moreover, for an $\alpha$ phase thin film with a large odd number of monolayers, it can be viewed as a $2 \mathrm{D} Z_{2} \mathrm{TI}$ with a helical edge state. Apparently, this edge state can only appear at hinges and form a loop, demonstrating that the $\alpha$ phase is a higher-order TI [21]. However, we do note that further direct evidence of the hinge states is desirable.

Fundamentally, our result implies that even without strong correlation and magnetism, there are still many topological materials that are beyond the scope of the already efficacious topological quantum chemistry or symmetry indicators [28-30]. Significantly, our work reveals the strong tunability of the quasi-1D TI $\mathrm{Bi}_{4} \mathrm{I}_{4}$ as a novel material platform where the abundance of topological phases could be explored. In the future, it would be exciting to investigate in this platform, for example, the strain-induced topological phases and their transitions, the spatial locations and Luttinger parameters of helical hinge states, the possible intrinsic and extrinsic topological superconductivity, as well as applications of our identified room-temperature topological phase transition as a topological switch that controls the dimensions of the boundary conduction channels.

\section{ACKNOWLEDGMENTS}

This work is mainly supported by National Science Foundation (NSF) through the DMREF program. This research used resources of the Stanford Synchrotron Radiation Lightsource and the Advanced Light Source, both U.S. DOE Office of Science User Facilities under Contracts No. AC02-76SF00515 and No. DE-AC0205CH112319, respectively. We acknowledge the Texas Advanced Computing Center (TACC) for providing resources that have contributed to the research results reported in this work. The work at Rice is supported by NSF under Grant No. DMR-1921847, the Gordon and Betty Moore Foundation's EPiQS Initiative through Grant No. GBMF9470, the Robert A. Welch Foundation under Grant No. C-2024, and the Sloan Foundation FG-201912224. The work at U.C.B. is supported by NSF Grant No. DMR-1921798. The work at U. T. D. is supported by NSF under Grants No. DMR-1921581 and 
No. DMR-1945351, AFOSR under Grant No. FA9550-191-0037, and Army Research Office (ARO) under Grant No. W911NF-18-1-0416. The work at O. S. U. is supported by NSF under Grant No. DMR-1922076.

[1] C. L. Kane and E. J. Mele, $\mathrm{Z}_{2}$ Topological Order and the Quantum Spin Hall Effect, Phys. Rev. Lett. 95, 146802 (2005).

[2] M. Konig, S. Wiedmann, C. Brune, A. Roth, H. Buhmann, L. W. Molenkamp, X.-L. Qi, and S.-C. Zhang, Quantum Spin Hall Insulator State in HgTe Quantum Wells, Science 318, 766 (2007).

[3] L. Fu, C. L. Kane, and E. J. Mele, Topological Insulators in Three Dimensions, Phys. Rev. Lett. 98, 106803 (2007).

[4] J. E. Moore and L. Balents, Topological Invariants of TimeReversal-Invariant Band Structures, Phys. Rev. B 75, 121306(R) (2007).

[5] M. Z. Hasan and C. L. Kane, Colloquium: Topological Insulators, Rev. Mod. Phys. 82, 3045 (2010).

[6] X.-L. Qi and S.-C. Zhang, Topological Insulators and Superconductors, Rev. Mod. Phys. 83, 1057 (2011).

[7] C.-C. Liu, J.-J. Zhou, Y. Yao, and F. Zhang, Weak Topological Insulators and Composite Weyl Semimetals: $\beta$ - $\mathrm{Bi}_{4} \mathrm{X}_{4}(X=\mathrm{Br}, I)$, Phys. Rev. Lett. 116, 066801 (2016).

[8] Y. Xia, D. Qian, D. Hsieh, L. Wray, A. Pal, H. Lin, A. Bansil, D. Grauer, Y. S. Hor, R. J. Cava, and M. Z. Hasan, Observation of a Large-Gap Topological-Insulator Class with a Single Dirac Cone on the Surface, Nat. Phys. 5, 398 (2009).

[9] H. Zhang, C.-X. Liu, X.-L. Qi, X. Dai, Z. Fang, and S.-C. Zhang, Topological Insulators in $\mathrm{Bi}_{2} \mathrm{Se}_{3}, \mathrm{Bi}_{2} \mathrm{Te}_{3}$ and $\mathrm{Sb}_{2} \mathrm{Te}_{3}$ with a Single Dirac Cone on the Surface, Nat. Phys. 5, 438 (2009).

[10] Y. L. Chen, J. G. Analytis, J.-H. Chu, Z. K. Liu, S.-K. Mo, X. L. Qi, H. J. Zhang, D. H. Lu, X. Dai, Z. Fang, S. C. Zhang, I. R. Fisher, Z. Hussain, and Z.-X. Shen, Experimental Realization of a Three-Dimensional Topological Insulator, $\mathrm{Bi}_{2} \mathrm{Te}_{3}$, Science 325, 178 (2009).

[11] D. Hsieh, Y. Xia, D. Qian, L. Wray, J. H. Dil, F. Meier, J. Osterwalder, L. Patthey, J. G. Checkelsky, N. P. Ong, A. V. Fedorov, H. Lin, A. Bansil, D. Grauer, Y. S. Hor, R. J. Cava, and M. Z. Hasan, A Tunable Topological Insulator in the Spin Helical Dirac Transport Regime, Nature (London) 460, 1101 (2009).

[12] B. Yan, L. Müchler, and C. Felser, Prediction of Weak Topological Insulators in Layered Semiconductors, Phys. Rev. Lett. 109, 116406 (2012).

[13] B. Rasche, A. Isaeva, M. Ruck, S. Borisenko, V. Zabolotnyy, B. Büchner, K. Koepernik, C. Ortix, M. Richter, and J. van den Brink, Stacked Topological Insulator Built from Bismuth-Based Graphene Sheet Analogues, Nat. Mater. 12, 422 (2013).

[14] P. Tang, B. Yan, W. Cao, S.-C. Wu, C. Felser, and W. Duan, Weak Topological Insulators Induced by the Interlayer Coupling: A First-Principles Study of Stacked $\mathrm{Bi}_{2}$ TeI, Phys. Rev. B 89, 041409(R) (2014).
[15] G. Yang, J. Liu, L. Fu, W. Duan, and C. Liu, Weak Topological Insulators in PbTe/SnTe Superlattices, Phys. Rev. B 89, 085312 (2014).

[16] X. Li, F. Zhang, Q. Niu, and J. Feng, Superlattice Valley Engineering for Designer Topological Insulators, Sci. Rep. 4, 6397 (2015).

[17] K. Lee, G. F. Lange, L.-L. Wang, B. Kuthanazhi, T. V. Trevisan, N. H. Jo, B. Schrunk, P. P. Orth, R.-J. Slager, P. C. Canfield, and A. Kaminski, Discovery of a Weak Topological Insulating State and van Hove Singularity in Triclinic $\mathrm{RhBi}_{2}$, Nat. Commun. 12, 1855 (2021).

[18] F. Zhang, C. L. Kane, and E. J. Mele, Surface State Magnetization and Chiral Edge States on Topological Insulators, Phys. Rev. Lett. 110, 046404 (2013).

[19] H. C. Po, A. Vishwanath, and H. Watanabe, SymmetryBased Indicators of Band Topology in the 230 Space Groups, Nat. Commun. 8, 50 (2017).

[20] F. Schindler, A. M. Cook, M. G. Vergniory, Z. Wang, S. S. P. Parkin, B. A. Bernevig, and T. Neupert, Higher-Order Topological Insulators, Sci. Adv. 4, eaat0346 (2018).

[21] C. Yoon, C.-C. Liu, H. Min, and F. Zhang, Quasi-OneDimensional Higher-Order Topological Insulators, arXiv: 2005.14710.

[22] H. Weng, X. Dai, and Z. Fang, Transition-Metal Pentatelluride $\mathrm{ZrTe}_{5}$ and $\mathrm{HfTe}_{5}$ : A Paradigm for Large-Gap Quantum Spin Hall Insulators, Phys. Rev. X 4, 011002 (2014).

[23] J. Mutch, W.-C. Chen, P. Went, T. Qian, I. Z. Wilson, A. Andreev, C.-C. Chen, and J.-H. Chu, Evidence for a StrainTuned Topological Phase Transition in $\mathrm{ZrTe}_{5}$, Sci. Adv. 5, eaav9771 (2019).

[24] P. Zhang, R. Noguchi, K. Kuroda, C. Lin, K. Kawaguchi, K. Yaji, A. Harasawa, M. Lippmaa, S. Nie, H. Weng, V. Kandyba, A. Giampietri, A. Barinov, Q. Li, G. D. Gu, S. Shin, and T. Kondo, Observation and Control of the Weak Topological Insulator State in $\mathrm{ZrTe}_{5}$, Nat. Commun. 12, 406 (2021).

[25] H. G. von Schnering, H. von Benda, and C. Kalveram, Wismutmonojodid BiJ, eine Verbindung mit $\mathrm{Bi}(\mathrm{O})$ und Bi(II), Z. Anorg. Allg. Chem. 438, 37 (1978).

[26] G. Autès, A. Isaeva, L. Moreschini, J. C. Johannsen, A. Pisoni, R. Mori, W. Zhang, T. G. Filatova, A. N. Kuznetsov, L. Forró, W. Van den Broek, Y. Kim, K. S. Kim, A. Lanzara, J. D. Denlinger, E. Rotenberg, A. Bostwick, M. Grioni, and O. V. Yazyev, A Novel Quasi-One-Dimensional Topological Insulator in Bismuth Iodide $\beta-\mathrm{Bi}_{4} \mathrm{I}_{4}$, Nat. Mater. 15, 154 (2016).

[27] R. Noguchi et al., A Weak Topological Insulator State in Quasi-One-Dimensional Bismuth Iodide, Nature (London) 566, 518 (2019).

[28] T. Zhang, Y. Jiang, Z. Song, H. Huang, Y. He, Z. Fang, H. Weng, and C. Fang, Catalogue of Topological Electronic Materials, Nature (London) 566, 475 (2019).

[29] M. G. Vergniory, L. Elcoro, C. Felser, N. Regnault, B. A. Bernevig, and Z. Wang, A Complete Catalogue of HighQuality Topological Materials, Nature (London) 566, 480 (2019).

[30] F. Tang, H. C. Po, A. Vishwanath, and X. Wan, Comprehensive Search for Topological Materials Using Symmetry Indicators, Nature (London) 566, 486 (2019). 
[31] B. I. Halperin, Possible States for a Three-Dimensional Electron Gas in a Strong Magnetic Field, Jpn. J. Appl. Phys. 26, 1913 (1987).

[32] F. Tang, Y. Ren, P. Wang, R. Zhong, J. Schneeloch, S. A. Yang, K. Yang, P. A. Lee, G. Gu, Z. Qiao, and L. Zhang, Three-Dimensional Quantum Hall Effect and Metalinsulator Transition in $\mathrm{ZrTe}_{5}$, Nature (London) 569, 537 (2019).

[33] See Supplemental Material at http://link.aps.org/ supplemental/10.1103/PhysRevX.11.031042 for details on experimental methods, sample characterization, computational methods, core level of $\mathrm{Bi}_{4} \mathrm{I}_{4}$, additional electronic structure measurements and calculations.

[34] W. P. Su, J. R. Schrieffer, and A. J. Heeger, Solitons in Polyacetylene, Phys. Rev. Lett. 42, 1698 (1979).

[35] Y. Tanaka, Z. Ren, T. Sato, K. Nakayama, S. Souma, T. Takahashi, K. Segawa, and Y. Ando, Experimental Realization of a Topological Crystalline Insulator in SnTe, Nat. Phys. 8, 800 (2012).

[36] S.-Y. Xu et al., Observation of a Topological Crystalline Insulator Phase and Topological Phase Transition in $\mathrm{Pb}_{1-x} \mathrm{Sn}_{x}$ Te, Nat. Commun. 3, 1192 (2012).
[37] Z. K. Liu, J. Jiang, B. Zhou, Z. J. Wang, Y. Zhang, H. M. Weng, D. Prabhakaran, S.-K. Mo, H. Peng, P. Dudin, T. Kim, M. Hoesch, Z. Fang, X. Dai, Z. X. Shen, D. L. Feng, Z. Hussain, and Y. L. Chen, A Stable Three-Dimensional Topological Dirac Semimetal $\mathrm{Cd}_{3} \mathrm{As}_{2}$, Nat. Mater. 13, 677 (2014).

[38] Z. K. Liu, B. Zhou, Y. Zhang, Z. J. Wang, H. M. Weng, D. Prabhakaran, S.-K. Mo, Z. X. Shen, Z. Fang, X. Dai, Z. Hussain, and Y. L. Chen, Discovery of a Three-Dimensional Topological Dirac Semimetal, $\mathrm{Na}_{3} \mathrm{Bi}$, Science 343, 864 (2014).

[39] S.-Y. Xu et al., Discovery of a Weyl Fermion Semimetal and Topological Fermi Arcs, Science 349, 613 (2015).

[40] B. Q. Lv, N. Xu, H. M. Weng, J. Z. Ma, P. Richard, X. C. Huang, L. X. Zhao, G. F. Chen, C. E. Matt, F. Bisti, V. N. Strocov, J. Mesot, Z. Fang, X. Dai, T. Qian, M. Shi, and H. Ding, Observation of Weyl Nodes in TaAs, Nat. Phys. 11, 724 (2015).

[41] B. Lv, T. Qian, and H. Ding, Angle-Resolved Photoemission Spectroscopy and Its Application to Topological Materials, Nat. Rev. Phys. 1, 609 (2019).

[42] J. A. Sobota, Y. He, and Z.-X. Shen, Angle-Resolved Photoemission Studies of Quantum Materials, Rev. Mod. Phys. 93, 025006 (2021). 